\title{
On The Dimensional Methods in Rare b Decays *
}

\author{
Mikołaj Misiak ${ }^{\dagger}$ \\ Physik-Department, \\ Technische Universität München, \\ 85748 Garching, FRG
}

August 1993

\begin{abstract}
Since several years there exists a question whether the dimensional reduction and the usual dimensional regularization give different results for the QCD-improved $b \rightarrow s \gamma$ and $b \rightarrow s$ gluon decay rates. Here it is demonstrated explicitly that this is not the case: As long as physically meaningful quantities are considered, the results obtained with help of both techniques agree.
\end{abstract}

TUM-T31-46/93

* Supported by the German Bundesministerium für Forschung und Technologie under contract 06 TM 732

${ }^{\dagger}$ On leave of absence from Institute of Theoretical Physics, Warsaw University. 


\section{Introduction.}

Since several years there exists a question whether the dimensional reduction (DRED) and the usual dimensional regularization with fully anticommuting $\gamma_{5}$ (NDR) give different results for the QCD-improved $b \rightarrow s \gamma$ and $b \rightarrow s$ gluon decay rates [1]. The purpose of this letter is to show explicitly that this is not the case: As long as physically meaningful quantities are considered, the results obtained with help of both methods agree.

The discussion presented here will be based only on the decay $b \rightarrow s \gamma$ which receives continuous interest at present, because of the recent measurement [2] of the $B \rightarrow K^{*} \gamma$ decay.

The $b \rightarrow s \gamma$ proceess at the leading order in the Standard Model interactions is given by the sum of the one-loop diagrams of Fig.1. The QCD contributions can be diagramatically represented by connecting the quark lines of these diagrams with an arbitrary number of gluon lines. The diagrams constructed this way form a power series in the parameter $\alpha_{Q C D}\left(M_{W}\right) \ln \left(M_{W}^{2} / m_{b}^{2}\right)$ $\simeq 0.7$ which seems too large to be an expansion parameter. Therefore one has to resum all the large logarithms with help of the Operator Product Expansion and the Renormalization Group Equations (RGE).

In order to do this, one introduces the local effective hamiltonian円

$$
H_{\text {eff }}=-\frac{4 G_{F}}{\sqrt{2}} V_{t s}^{*} V_{t b}\left[\sum_{i=1}^{n} c_{i}(\mu) O_{i}+\text { counterterms }\right]
$$

A complete list of the operators $O_{i}$ is given in each of the papers [3, 5, 6, 6, 7, \&]. As pointed out in ref. [5], the papers [1, 4 do not include all the operators relevant in the leading-logarithmic approximation. This problem is, however, to a large extent unrelated to the problem whether both dimensional methods give the same results.

For the purpose of this paper, it is enough to give explicitly now only three of the operators $O_{i}$.

$$
\begin{gathered}
O_{2}=\left(\bar{s}_{L} \gamma_{\mu} c_{L}\right)\left(\bar{c}_{L} \gamma^{\mu} b_{L}\right) \\
O_{7}=\frac{e}{16 \pi^{2}} m_{b} \bar{s}_{L} \sigma_{\mu \nu} F^{\mu \nu} b_{R} \\
O_{8}=\frac{g}{16 \pi^{2}} m_{b} \bar{s}_{L} \sigma_{\mu \nu} G^{\mu \nu} b_{R}
\end{gathered}
$$

where $F^{\mu \nu}$ and $G^{\mu \nu}$ are the photonic and gluonic field strenght tensors, respectively. One finds the coefficients of these operators by requireing equality of amplitudes generated by the effective Hamiltonian (1) and the full

\footnotetext{
${ }^{1}$ For simplicity we neglect the small $V_{u s}^{*} V_{u b}$ in our discussion. However, the basic results summarized in eqs. (7), (8) and in the Appendix are exactly the same even if $V_{u s}^{*} V_{u b}$ is not neglected.
} 
Standard Model amplitudes (up to $0\left(1 / M_{W}^{4}\right)$ ) at the renormalization scale $\mu=M_{W}$. The well-known results are:

$$
\begin{gathered}
c_{2}\left(M_{W}\right)=1 \\
c_{7}\left(M_{W}\right)=\frac{3 x^{3}-2 x^{2}}{4(x-1)^{4}} \ln (x)+\frac{-8 x^{3}-5 x^{2}+7 x}{24(x-1)^{3}} \\
c_{8}\left(M_{W}\right)=\frac{-3 x^{2}}{4(x-1)^{4}} \ln (x)+\frac{-x^{3}+5 x^{2}+2 x}{8(x-1)^{3}}
\end{gathered}
$$

Here $x=m_{t}^{2} / M_{W}^{2}$. The above coefficients are regularization- and renormalization-scheme independent. In order to avoid appearance of large logarithms in the $b \rightarrow s \gamma$ matrix element of $H_{\text {eff }}$ (1), one evolves the coefficients $c_{i}(\mu)$ down to the scale $\mu=m_{b}$, according to the RGE:

$$
\mu \frac{d}{d \mu} c_{i}(\mu)-\sum_{i=1}^{n} \gamma_{j i}\left(\alpha_{Q C D}\right) c_{j}(\mu)=0
$$

Finally, one finds that the $b \rightarrow s \gamma$ matrix element of $H_{\text {eff }}$ (1) evaluated at $\mu=m_{b}$ (in the leading-logarithmic approximation and for the photon on shell) is equal to the tree-level matrix element of F:

$$
-\frac{4 G_{F}}{\sqrt{2}} V_{t s}^{*} V_{t b} c_{7}^{e f f}\left(m_{b}\right) O_{7}
$$

with

$$
c_{7}^{e f f}\left(m_{b}\right)=\eta^{\frac{16}{23}} c_{7}\left(M_{W}\right)+\frac{8}{3}\left(\eta^{\frac{14}{23}}-\eta^{\frac{16}{23}}\right) c_{8}\left(M_{W}\right)+c_{2}\left(M_{W}\right) \sum_{i=1}^{8} a_{i} \eta^{b_{i}}
$$

where $\eta=\frac{\alpha\left(M_{W}\right)}{\alpha\left(m_{b}\right)}$, and $a_{i}, b_{i}$ are some exact numbers that should be regularizationand renormalization-scheme independent.

The form of the last expression agrees with the final results of any of the papers [1, 3, 4, 5, 6, 7, 8. However, the particular values of the numbers $a_{i}$ disagree for any two of them. The numbers $a_{i}$ and $b_{i}$ are given and discussed in the Appendix.

In order to make our discussion as simple as possible, we formally expand the last term in (8) in powers of $\alpha_{Q C D}\left(M_{W}\right)$ :

$$
\sum_{i=1}^{8} a_{i} \eta^{b_{i}}=-X \frac{\alpha_{Q C D}\left(M_{W}\right)}{2 \pi} \ln \frac{M_{W}}{m_{b}}+0\left[\left(\alpha_{Q C D}\left(M_{W}\right) \ln \frac{M_{W}}{m_{b}}\right)^{2}\right]
$$

where

$$
X=\frac{23}{3} \sum_{i=1}^{8} a_{i} b_{i}
$$

\footnotetext{
${ }^{2}$ The s-quark mass is neglected throughout.
} 
From the NDR results of refs. [9, 3, 1, 4, 5, 6, 7, 8] it follows that $X=$ $\frac{8}{3}, \frac{232}{81}, \frac{232}{81}, \frac{232}{81}, \frac{208}{81}, \frac{208}{81}, \frac{208}{81}, \frac{208}{81}$, respectively. The first change (from $\frac{8}{3}$ to $\frac{232}{81}$ ) is due to the inclusion of the contributions proportional to the down-quark charge, that had not been included in the first paper. The second change (from $\frac{232}{81}$ to $\frac{208}{81}$ ) is due to taking into account certain one-loop matrix elements which will be described in the next section. The value of $\frac{208}{81}$ has been very recently confirmed [8] with help of the HV scheme [10] where no one-loop matrix elements enter. The only known DRED calculation (see ref. [1]) gives $\mathrm{X}=\frac{124}{81}$. In the following two sections, the calculation of $\mathrm{X}$ in NDR and DRED will be presented and shown to give the same result of $\frac{208}{81}$. We restrict ourselves only to the quantity $\mathrm{X}$ (which has been the subject of the main discussion in the past) in order to avoid considering all the subtleties involved in the calculation of the remaining terms in the r.h.s. of eq. (9) where most of the disagreements between the existing NDR calculations are located (see the Appendix).

\section{The NDR calculation of X.}

In order to calculate $\mathrm{X}$, we have to trace out all possible leading-logarithmic contributions to $<s \gamma\left|H_{e f f}\right| b>_{\mu=m_{b}}$ that are proportional to $c_{2}\left(M_{W}\right)$. Similarly to refs. [1, 4], and differently than in refs. [3, 5, 6, 7, 8], we will perform the calculation without applying the equations of motion to the operators we encounter.

First of all, we have to consider all possible divergent one-loop 1PI diagrams with the $\mathrm{O}_{2}$-vertex, except for those that contain more than one power of the QED-coupling $e$. There are four types of such diagrams, presented on Figs. 2a-2d, respectively.

The diagram in Fig.2a vanishes for the on-shell photon. This is why there is no one-loop mixing between $\mathrm{O}_{2}$ and $O_{7}$. In effect, the two-loop mixing between these two operators becomes important in the leading logarithmic approximation.

All the diagrams in Fig.2b require only counterterms proportional to the four-quark operators containing the $\bar{c}_{L}$ and $c_{L}$ fields. None of such operators can have nonvanishing one-loop $b \rightarrow s \gamma$ matrix elements for the on-shell photon. Consequently, these diagrams are irrelevant in the calculation of $\mathrm{X}$.

The diagrams in Fig.2c generate no divergencies in NDR.

The divergencies generated by the diagrams in Fig.2d can be exactly

\footnotetext{
${ }^{3}$ In this scheme $\gamma_{5}$ anticommutes with the 4-dimensional $\gamma_{\mu}$ 's, but commutes with the remaining ones. It is the only known scheme where problems with traces containing odd numbers of $\gamma_{5}$ 's do not appear. The treatment of $\gamma_{5}$ is the only difference between the HV and NDR schemes.
} 
cancelled by the counterterm (see eq.(1)):

$$
c_{2}(\mu) Z_{2 G} O_{G}
$$

where

$$
O_{G}=\frac{g}{16 \pi^{2}} \bar{s}_{L} \gamma_{\mu}\left(D_{\nu} G^{\mu \nu}\right) b_{L}
$$

and

$$
Z_{2 G}=-\frac{4}{3(4-d)}+(\text { terms finite in the limit } d \rightarrow 4)
$$

From $Z_{2 G}$ we recover the corresponding element of the anomalous dimension matrix

$$
\gamma_{2 G}=-\frac{4}{3}+0\left(\alpha_{Q C D}\right)
$$

and immediately find the relevant term in the solution of the RGE (6) for $c_{G}(\mu)$

$$
c_{G}(\mu)=c_{G}\left(M_{W}\right)+\frac{4}{3} c_{2}\left(M_{W}\right) \ln \frac{M_{W}}{\mu}+0\left(\alpha_{Q C D} \ln \frac{M_{W}}{\mu}\right)
$$

We could write all the remaining terms as $0\left(\alpha_{Q C D} \ln \frac{M_{W}}{\mu}\right)$ because there is no other than $\mathrm{O}_{2}$ operator that has a coefficient of order 1 at $\mu=M_{W}$ and mixes with $O_{G}$ at zeroth orderf in $\alpha_{Q C D}$.

Now, let us consider the one-loop on-shell matrix element of $O_{G}$. It is given by the diagrams in Fig.3. When a matrix element is considered, we have to take into account also the $1 \mathrm{PR}$ diagrams. The sum of the diagrams in Fig.3 appears to be proportional to the tree-level matrix element of $\mathrm{O}_{7}$ :

$$
\left(\begin{array}{c}
\text { Sum of the diagrams } \\
\text { in Fig.3 at } \mu=m_{b}
\end{array}\right)_{\text {on shell }}=-\frac{4 G_{F}}{\sqrt{2}} V_{t s}^{*} V_{t b} c_{G}\left(m_{b}\right) \frac{\alpha_{Q C D}}{2 \pi} \frac{2}{9}<s \gamma\left|O_{7}\right| b>
$$

Comparing eqs. (7), (8), (9), (15) and (16) one finds the contribution to $\mathrm{X}$ from the one-loop matrix element of $O_{G}$ :

$$
\Delta X_{\text {matrix element }}=-\frac{8}{27}
$$

A contribution to $c_{7}^{e f f}$ from one-loop matrix elements has been already found in ref. [5]. As mentioned there, its particular value (that corresponds to $-\frac{8}{27}$ above) is correct only in dimensional regularization with fully anticommuting $\gamma_{5}$. In any 4-dimensional scheme (or in the HV scheme) the on-shell $b \rightarrow s \gamma$ one-loop matrix element of $O_{G}$ vanishes. But the contribu-

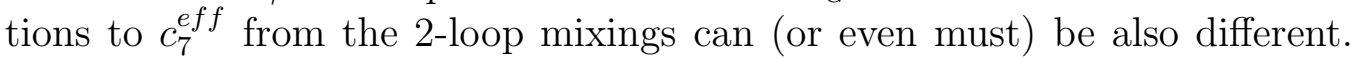
This point has been recently emphasized in ref. [8].

\footnotetext{
${ }^{4}$ in the applied normalization for $O_{G}$
} 
What remains to be considered is the two-loop mixing $\mathrm{O}_{2} \rightarrow O_{7}$. It is described by the diagrams in Fig.4. The calculation of these diagrams is described in great detail in ref. [3]. The well-known result is

$$
\gamma_{27}=\frac{\alpha_{Q C D}}{2 \pi} \frac{232}{81}
$$

After solving the RGE of eq.(6) ) and formally expanding the solution in $\alpha_{Q C D}$ we immediately get

$$
\Delta X_{\text {mixing }}=\frac{232}{81}
$$

Therefore, the final value of $\mathrm{X}$ is

$$
X=\Delta X_{\text {matrix element }}+\Delta X_{\text {mixing }}=-\frac{8}{27}+\frac{232}{81}=\frac{208}{81}
$$

\section{The DRED calculation of $\mathrm{X}$.}

The DRED scheme has been introduced by Siegel 11] in order to be able to dimensionally regularize supersymmetric theories without actually breaking supersymmetry. The only difference between NDR and DRED is that in the latter scheme all the tensor fields are left 4-dimensional, while the momenta and coordinates are d-dimensional. The dimension $\mathrm{d}$ is assumed to be "smaller" than 4, which means that in practical calculations one applies the equalityf:

$$
g_{\mu \nu}^{(4)} g^{\nu \rho}=g_{\mu}{ }^{\rho}
$$

where $g_{\mu \nu}^{(4)}$ and $g^{\mu \nu}$ denote the 4-dimensional and the d-dimensional metric tensors, respectively. As it is implicit in the above equation, we allow the indices of the d-dimensional tensors to acquire values also larger than $\mathrm{d}$, but then the corresponding components of these tensors are assumed to vanish.

For clarity, we will keep the superscript "(4)" for all the 4-dimensional tensors that appear in this section. It is also necessary to introduce some notation for the diference between the 4-dimensional and the d-dimensional tensors. We define:

$$
G_{\mu}^{(\epsilon)}=G_{\mu}^{(4)}-G_{\mu}
$$

for the gluonic field, and

$$
\gamma_{\mu}^{(\epsilon)}=\gamma_{\mu}^{(4)}-\gamma_{\mu}
$$

for the Dirac matrices. The matrix $\gamma_{5}$ is taken to be anticommuting with all the $\gamma_{\mu}^{(4)}$ 's and $\gamma_{\mu}$ 's 11].

We should supply the superscripts "(4)" for all the fields and Dirac matrices in the definitions of the three operators we started with in eq. (2), and

\footnotetext{
${ }^{5}$ for arbitrary values of all the other mixings

${ }^{6}$ opposite to the HV scheme where $g_{\mu \nu}^{(4)} g^{\nu \rho}=g_{\mu}^{(4) \rho}$ is used
} 
to the operator in eq. (12). The "new" operators will be denoted by $\mathrm{O}_{2}^{(4)}$, $O_{7}^{(4)}, O_{8}^{(4)}$ and $O_{G}^{(4)}$, respectively.

We proceed along the same lines as in the previous section. We have to consider the divergent parts of the diagrams in Figs. 2a-2d, but now with the $\mathrm{O}_{2}^{(4)}$-vertex. The diagrams of Figs. $2 \mathrm{a}$ and $2 \mathrm{~b}$ are elliminated from our discussion with help of the same arguements as in the NDR case.

The first real difference between NDR and DRED is in the case of the diagrams in Fig. 2c. In the DRED case the sum of the diagrams with one gluon and one photon does not vanish, but gives a divergence proportional to the operator:

$$
O_{x}=\frac{e g}{16 \pi^{2}} \bar{s}_{L}^{(4)} \sigma_{\mu \nu}^{(4)} F^{(4) \mu \nu} \gamma_{\rho}^{(\epsilon)} G^{(\epsilon) \rho} b_{L}^{(4)}
$$

The required renormalization constant is

$$
Z_{2 x}=+\frac{4}{3(4-d)}+(\text { terms finite in the limit } d \rightarrow 4)
$$

So, similarly to the case of $O_{G}$ discussed in the previous section, we get (cf. eqs. (11)-(15)):

$$
c_{x}(\mu)=c_{x}\left(M_{W}\right)-\frac{4}{3} c_{2}\left(M_{W}\right) \ln \frac{M_{W}}{\mu}+0\left(\alpha_{Q C D} \ln \frac{M_{W}}{\mu}\right)
$$

The diagrams in Fig.2c that contain two gluons are irrelevant in the calculation of X, because the one-loop $b \rightarrow s \gamma$ matrix element of an operator containing two gluons is of order $\alpha_{Q C D}^{2}$.

The diagrams in Fig.2d generate two important counterterms. One of them is, of course, the counterterm proportional to $O_{G}^{(4)}$ with the same renormalization constant as in eq. (13). The other is proportional to the operator

$$
O_{y}=\frac{g}{16 \pi^{2}} \bar{s}_{L}^{(4)} \gamma_{\mu}^{(\epsilon)} \square G^{(\epsilon) \mu} b_{L}^{(4)}
$$

with the renormalization constant

$$
Z_{2 y}=+\frac{2}{3(4-d)}+(\text { terms finite in the limit } d \rightarrow 4)
$$

So, the coefficient of $O_{y}$ behaves like

$$
c_{y}(\mu)=c_{y}\left(M_{W}\right)-\frac{2}{3} c_{2}\left(M_{W}\right) \ln \frac{M_{W}}{\mu}+0\left(\alpha_{Q C D} \ln \frac{M_{W}}{\mu}\right)
$$

The diagrams containing more than one gluon in Fig.2d may also give rise to some other counterterms containing the $G_{\mu}^{(\epsilon)}$ field. They are, however, irrelevant for the same reason as the two-gluon diagrams in Fig.2c. 
The appearance of the counterterms involving the $G_{\mu}^{(\epsilon)}$ that break gauge invariance in the $(4-d)$-dimensional subspace is nothing surprising in DRED. They have been observed already by the inventor of DRED [11].

Now, let us consider the one-loop on-shell matrix elements of the operators $O_{x}, O_{G}^{(4)}$ and $O_{y}$. The first of them is given by the sum of diagrams in Fig.5. The sum of these diagrams appears to be proportional to the tree level matrix element of $O_{7}^{(4)}$ (we ignore the possible terms proportional to the $(4-d)$-dimensional photonic field):

$$
\left(\begin{array}{c}
\text { Sum of the diagrams } \\
\text { in Fig.5 at } \mu=m_{b}
\end{array}\right)_{\text {on shell }}=-\frac{4 G_{F}}{\sqrt{2}} V_{t s}^{*} V_{t b} c_{x}\left(m_{b}\right) \frac{\alpha_{Q C D}}{2 \pi}\left(-\frac{2}{3}\right)<s \gamma\left|O_{7}\right| b>
$$

Comparing eqs. (7), (8), (9), (26) and (30) one finds:

$$
\Delta X_{\text {matrix element of } O_{x}}=-\frac{8}{9}
$$

The one-loop matrix elements of $O_{G}^{(4)}$ and $O_{y}$ are described by the same diagrams as in the case of $O_{G}$ (Fig.3) - we only have to change the operator vertex. In the case of $O_{G}^{(4)}$ the sum of these diagrams vanishes on-shell, while in the case of $O_{y}$ we get exactly the same as in eq. (16). The correlation is not surprising, because

$$
O_{G}=O_{G}^{(4)}+O_{y}
$$

Using these results in the same way as before, we get:

$$
\Delta X_{\text {matrix element of } O_{G}^{(4)}}=0 \quad \Delta X_{\text {matrix element of } O_{y}}=+\frac{4}{27}
$$

So the sum of the contributions to $\mathrm{X}$ from the one-loop matrix elements is

$$
\Delta X_{\text {matrix elements }}=-\frac{8}{9}+0+\frac{4}{27}=-\frac{20}{27}
$$

Now, we have to consider the two-loop mixing $O_{2}^{(4)} \rightarrow O_{7}^{(4)}$. The twoloop diagrams look exactly the same as in the NDR case (Fig.4). In the one-loop counterterm diagrams of Fig. 4 we have to insert both the $O_{G}^{(4)}$ - and the $O_{y}$-counterterms. Finally, we have to take into account also the one-loop counterterm diagrams with the counterterms proportional to $O_{x}$. The latter diagrams can be obtained from the ones in Fig.5 just by replacing the square (representing the $O_{x}$-vertex) by a cross (representing the $O_{x}$-counterterm).

The details of the two-loop calculation will not be presented here. It has been done with help of the method used in ref. [13], i.e. only the difference 
between DRED and NDR has been calculated. Then one needs to consider only the double-pole parts of the two-loop integrals (given in ref. [3]), and also the Dirac algebra is relatively simpler.

The final result for the sought element of the anomalous-dimension matrix is:

$$
\gamma_{27}=\frac{\alpha_{Q C D}}{2 \pi}\left(\frac{124}{81}+\frac{16}{9}\right)=\frac{\alpha_{Q C D}}{2 \pi} \frac{268}{81}
$$

The number $\frac{16}{9}$ comes from the $O_{x}$-counterterm diagrams. This will be exactly the contribution from these diagrams to $\mathrm{X}$. It is not an accident that it equals to -2 (contribution to $\mathrm{X}$ from the one-loop matrix element of $O_{x}$ ). This is a common feature for the so-called "evanescent operators" i.e. operators vanishing in the limit $d \rightarrow 4$ (see e.g. refs. [14, [15, 6]).

The number $\frac{124}{81}$ in eq.(35) comes from the diagrams of Fig.4. It is in agreement with the findings of ref. [1], where the $O_{x}$ counterterms have not been included. The presence of the $O_{x}$ counterterms is also the reason why the tests made in ref. [12] did not work in the DRED case.

As in the NDR case, we recover the contribution to $\mathrm{X}$ from $\gamma_{27}$.

$$
\Delta X_{\text {mixing }}=\frac{268}{81}
$$

and we add it to the contribution from the matrix elements, to obtain the final result

$$
X=\Delta X_{\text {matrix elements }}+\Delta X_{\text {mixing }}=-\frac{20}{27}+\frac{268}{81}=\frac{208}{81}
$$

which is in agreement with the NDR result.

\section{Final remarks}

The following table summarizes the results for the quantity X obtained with help of the NDR, HV and DRED schemes.

\begin{tabular}{|c|c|c|c|}
\hline scheme & $\Delta X_{\text {matrix elements }}$ & $\Delta X_{\text {mixing }}$ & $X$ \\
\hline \hline NDR & $-\frac{8}{27}$ & $\frac{232}{81}$ & $\frac{208}{81}$ \\
\hline HV & 0 & $\frac{208}{81}$ & $\frac{208}{81}$ \\
\hline DRED & $-\frac{20}{27}$ & $\frac{268}{81}$ & $\frac{208}{81}$ \\
\hline
\end{tabular}

The result for $\Delta X_{\text {mixing }}$ in the HV scheme has been taken from eq.(25) of ref. [8]. 
The equality of all the three results for the physically meaningful quantity $\mathrm{X}$ is what one would naturally expect, assuming that all the three schemes are the consistent ones. This is also what one could expect by remembering the two-loop calculation of ref. [15] where all the three schemes were found to give the same results for the physically meaningful quantities in the fourquark operator case.

A scheme for extending the Dirac algebra to d-dimensions is consistent if it gives a proper limit at $d \rightarrow 4$ and is unique $?$. The latter requirement follows from the fact that a consistent regularization procedure must give the same results for a given diagram independently on whether it is considered separately or as a subdiagram, and independently on the order in which the subdiagrams are calculated.

By analyzing how all the three schemes are defined, one can realize that all of them are consistent as long as traces with odd numbers of $\gamma_{5}$ 's do not appear. If they appear, then only the HV scheme remains consistent. In the two other schemes we find that the expressions like

$$
\operatorname{Tr}\left(\gamma_{\alpha} \gamma_{\mu} \gamma_{\nu} \gamma_{\rho} \gamma_{\sigma} \gamma_{5} \gamma_{\alpha}\right)
$$

in NDR, or

$$
\operatorname{Tr}\left(\gamma_{\alpha}^{(\epsilon)} \gamma_{\mu}^{(4)} \gamma_{\nu}^{(4)} \gamma_{\rho}^{(4)} \gamma_{\sigma}^{(4)} \gamma_{5} \gamma_{\alpha}^{(\epsilon)}\right)
$$

in DRED give different results dependently on whether the cyclicity of the trace is used, or the contracted $\gamma$ 's are commuted all the way through what stands between them.

In the calculation of X presented in this paper, as well as in the calculation of ref. [15] no traces appeared. This is why no discrepancies between the three schemes were observed.

Things become more complicated in the complete leading-logarithmic calculation of the $b \rightarrow s \gamma$ rate. In that case one has to use more refined arguements to show that the (sufficiently careful) NDR calculation is a consistent one. Two independent ways of such an argumentation have been given in the Appendix A of ref. [6]. The first was based on the idea of introducing certain "evanescent operators" in order to avoid specifying the algebraic properties of $\gamma_{5}$ before arriving at expressions that cannot lead to inconsistencies 8 . The other method was based on the observation, that certain symmetries in the

\footnotetext{
${ }^{7}$ We do not require that our regularization scheme preserves the symmetries of the theory. In the absence of anomalies, all the nonsymmetric terms are local and can be removed by proper nonsymmetric counterterms.

${ }^{8}$ This method is a consistent one, but in a general multi-loop calculation it is expected to be much more complicated than the usual HV scheme. However, in some particular calculations (especially such where no more than two $\gamma_{5}$ 's can appear in a single fermionic line) it can be much simpler.
} 
structure of all the relevant four-quark operators allows to perform the calculation with no need to calculate any traces. This observation has been also independently made in ref.[16], in the context of calculating similar diagrams for the next-to-leading effects in the $\Delta S=1$ transitions.

Both these methods of dealing with the dangerous traces can be directly used also in the DRED case. This is why one can expect that the sufficiently careful DRED calculation of all the numbers $a_{i}$ and $b_{i}$ from eq.(8) will give the same results as in the NDR and HV schemes ${ }^{\text {f }}$.

The author would like to thank Professor A. Buras for stimulating discussions.

\section{Appendix}

As mentioned below eq.(8), the numbers $a_{i}$ and $b_{i}$ present in this equation are subject to disagreements between any two of the existing calculations of

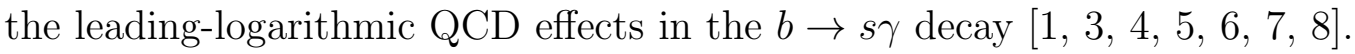
In most cases it is due to the fact that not all the relevant dimension-six operators are included. The complete basis of the operators (reduced by the equations of motion) has been written down already in ref. [3]. But the effects of the operators called $\mathbb{O}_{3}, \mathrm{O}_{4}, \mathrm{O}_{5}, \mathrm{O}_{6}$ have been neglected there.

The three most recent papers [6, 7, 8, where the effects of all the relevant operators are explicitly calculated, disagree on the mixings $\left(\mathrm{O}_{5}, \mathrm{O}_{6}\right) \rightarrow$ $\left(\mathrm{O}_{7}, \mathrm{O}_{8}\right)$. The authors of refs. [6] and [7] have performed a comparison of their calculations [17], and they have found two (and only two) sources of the disagreement

(i) not including the effects of the so-called "evanescent operators" in ref. [7]

(ii) disagreements in these parts of the first four two-loop diagrams in Fig.5 of ref. [6], where the fermion mass comes from the fermionic loop.

After arriving at this conclusion, we have received the paper [8]. The results of this paper can be reproduced, if the disagreement (i) is resolved in favour of ref. [6], and the disagreement (ii) - in favour of ref.[7]. However, none of the authors of the considered papers is ready to say at the moment that his previous results should be corrected. This is why I have decided to give here the numbers $a_{i}$ corresponding to each of the three papers [6, 0, 8]. They are as follows

\footnotetext{
${ }^{9}$ This is also the expectation of the authors of ref. [8] who declare to be just performing such a complete DRED calculation.

${ }^{10}$ The numbering of the operators common for refs. [3, 6, 6, 8] is used here.
} 
ref. [6]:

$a_{i}=\left(\frac{422534}{272277},-\frac{35533}{51730},-0.4286,-0.0714,-0.1991,-0.0453,-0.0215,-0.0990\right)$

ref. [7]:

$a_{i}=\left(\frac{708542}{272277},-\frac{69049}{51730},-0.4286,-0.0714,-0.7415,-0.0003,-0.0580,+0.0323\right)$

ref. 88]:

$a_{i}=\left(\frac{626126}{272277},-\frac{56281}{51730},-0.4286,-0.0714,-0.6494,-0.0380,-0.0186,-0.0057\right)$

The corresponding numbers $b_{i}$ are the following

$$
b_{i}=\left(\frac{14}{23}, \frac{16}{23}, \frac{6}{23},-\frac{12}{23}, 0.4086,-0.4230,-0.8994,0.1456\right)
$$

The numbers $b_{i}$ are insensitive to the disagreements between the papers [6, 7, 8]. This is because they are proportional to the eigenvalues of the blocktriangular anomalous-dimension matrices which disagree with each other only in the off-diagonal block. The latter six of the numbers $b_{i}$ are proportional to the eigenvalues of the anomalous dimension matrix for the $O_{1}-O_{6}$ oparators that has been calculated long ago in ref. 18]. The first two are given by the self-mixing of the $O_{7}$ and $O_{8}$ operators that has been originally found in ref. 19.

Some of the numbers $a_{i}$ and $b_{i}$ are not rational, but they are known to arbitrary precision because they come from the diagonalization of the leading-order anomalous-dimension matrices that are known exactly.

As it has been mentioned below eq.(8), the sum of all the numbers $a_{i}$ always vanishes. This is because all the QCD effects summarized in eq.(8) must vanish for $\eta=1$.

The numbers $a_{i}$ corresponding to the results of refs. [6] and [8] look very

much different. However, the difference between the resulting $c_{7}^{\text {eff }}$ 's is below $1 \%$. This can be easily undestood, because the differences between the anomalous dimension matrices found in these papers are only in the mixings $\mathrm{O}_{5} \rightarrow \mathrm{O}_{7}$ and $\left(\mathrm{O}_{5}, \mathrm{O}_{6}\right) \rightarrow \mathrm{O}_{8}$. The operator $\mathrm{O}_{5}$ acquires only a very small coefficient $(\sim 0.008)$ during the evolution from $M_{W}$ to $m_{b}$. The operator $O_{6}$ has a larger coefficient, but the effects of the mixing $O_{6} \rightarrow O_{8} \rightarrow O_{7}$ tend to cancel in the latter step (see the term proportional to $O_{8}$ in eq.(8)).

\section{References}

[1] R. Grigjanis, P.J. O'Donnell, M. Sutherland and H. Navelet, Phys. Lett. B213 (1988) 355 (see also Phys. Rept. 228 (1993) 93 and references therein)

[2] R. Ammar et. al., Phys. Rev. Lett. 71 (1993) 674

[3] B. Grinstein, R. Springer and M.B. Wise, Phys. Lett. B202 (1988) 138, Nucl. Phys. B339 (1990) 269 
[4] G. Cella, G. Curci, G. Ricciardi and A. Vicere, Phys. Lett. B248 (1990) 181

[5] M. Misiak, Phys. Lett. B269 (1991) 161

[6] M. Misiak, Nucl. Phys. B393 (1993) 23

[7] K. Adel and Y.P. Yao, Modern Physics Letters A8 (1993) 1679

[8] M. Ciuchini, E. Franco, G. Martinelli, L. Reina and L. Silvestrini preprint ROME 93/958 (hep-ph 9307364)

[9] S. Bertolini, F. Borzumati and A. Masiero, Phys. Rev. Lett. 59 (1987) 180

[10] G. 't Hooft and M. Veltman, Nucl. Phys. B44 (1972) 189

P. Breitenlohner and D. Maison, Commun. Math. Phys. 52 (1977) 11, 39, 55

[11] W. Siegel, Phys. Lett. B84 (1989) 193

[12] R. Grigjanis, P.J. O'Donnell, M. Sutherland and H. Navelet, Phys. Lett. B237 (1990) 252

[13] P. J. O'Donnell and H. K. K. Tung, Phys. Rev. D45 (1992) 4342

[14] M.J. Dugan and B. Grinstein, Phys. Lett. B256 (1991) 239

[15] A.J. Buras and P.H. Weisz, Nucl. Phys. B333 (1990) 66

[16] A.J. Buras, M. Jamin, M.E. Lautenbacher and P.H. Weisz, Nucl. Phys. B400 (1993) 37

[17] K. Adel, private communication

[18] F.J. Gilman and M.B. Wise, Phys. Rev. D20 (1979) 2392

[19] M.A. Shifman, A.I. Vainstein, V.I. Zakharov, Phys. Rev. D18 (1978) 2583

\section{Figure captions}

Fig 1. Diagrams contributing to $b \rightarrow s \gamma$ at the leading order of the SM interactions

Fig 2a-2d. One-loop divergent diagrams with the $\mathrm{O}_{2}$ vertex 
Fig 3. Diagrams contributing to the one-loop on-shell matrix element of $O_{G}, O_{G}^{(4)}$ or $O_{y}$. The square denotes the insertion of any of these three operators.

Fig 4. Diagrams contributing to the two-loop mixing of $O_{2}$ with $O_{7}$

Fig 5. Diagrams contributing to the one-loop matrix element of $O_{x}$ 


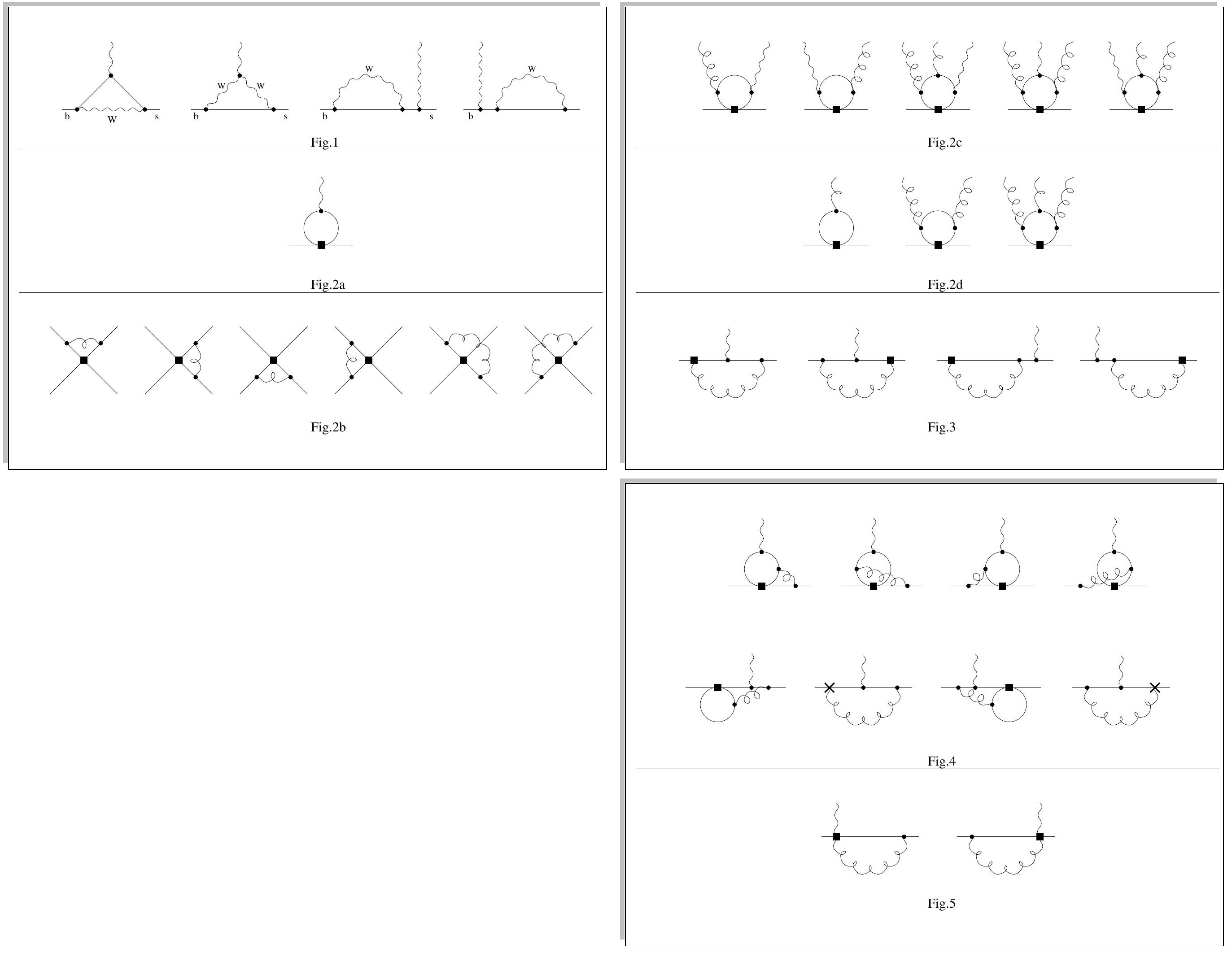

